\title{
Healthy lottery. An economically viable mobile system to increase compliance of individuals with diabetes
}

\author{
Riccardo Bonazzi ${ }^{1}$, Francesco Cimmino ${ }^{1}$, Katherine Blondon ${ }^{2}$ \\ ${ }^{1}$ University of Applied Sciences Western Switzerland, ${ }^{2}$ University Hospitals of Geneva \\ riccardo.bonazzi@hevs.ch, francesco.cimmino@hevs.ch, katherine.blondon@hcuge.ch
}

\begin{abstract}
This article shows the preliminary results of an ongoing study to develop an economically sustainable system, which financially rewards individuals with diabetes. Previous studies have already shown that monetary incentives appear to be the strongest motivator for older individuals with type II diabetes. Nonetheless, design criteria for a mobile service are not well established and there is no study available to assess the viability of a system that rewards individuals for self-management. Therefore, in this paper we explore a design theory, which describes a new mobile service that integrates data from other smartphone applications to assess therapeutic compliance. Our software includes a self-supported lottery in a business model, which allows patients with effective selfmanagement to be rewarded without any deficit. Our prototype is based on a social business model, which aims at improving patients' health and that can be described as "healthy" for them.
\end{abstract}

\section{Introduction}

This article is addressed to designers of diabetes management software, and more broadly to patients affected by type II diabetes and to their healthcare providers.

We are interested in diabetes management software on smartphones or tablets, which helps persons with type II diabetes manage the data associated with: (a) blood test results from a glucose meter, (b) log entries for exercise and other factors coming from pervasive systems, (c) coaching for dose corrections. Although there is a plethora of websites and mobile applications (also known as m-Health apps) for individuals with type 2 diabetes, there is a scarcity of reliable data concerning their added value for older patients [10]. A recent review of internet-based interventions to promote lifestyle modification among adults with type II diabetes found that (a) successful studies had interactive components with tracking and personalized feedback and opportunities for peer support, (b) website utilization declined over time in all studies and concluded that future research is needed on the engagement of patients over time [7]. In fact, the successful use and potential health benefits related to the electronic devices seems to depend more on the design of the engagement strategies than on the features of their technology [18]. To be effective, technologies must be paired with approaches that create and maintain engagement [25]. Hence, we sought a solution to improve self-management of older patients with type II diabetes, using mobile technologies and incentives to guide and maintain long-term engagement. Therefore, we introduce the notion of therapeutic compliance (hereinafter referred to as compliance) as patient's behaviors (in terms of taking medication, following diets, or executing life style changes) that coincide with healthcare providers' recommendations for health and medical advice [15,24]. Lack of compliance leads to multiple hospitalizations of patients and that significantly increases healthcare costs. A study of more than 600000 patients with diabetes showed that among those who had been hospitalized, $30 \%$ had two or more stays accounting for $50 \%$ of total hospitalizations and hospital costs [14]. Thus, we look for a solution to reduce those costs. A recent review of diabetes apps for iOS and Android operating systems examined whether the available applications serve the special needs of diabetes patients aged 50 or older by performing an expert-based usability evaluation. The authors suggested that (a) patients and physicians alike should be involved in the app development process to a greater extent, and that (b) the usability of diabetes apps for patients aged 50 or older was moderate to good, but this result applied mainly to apps offering a small range of functions [1]. Another study demonstrated that mobile phone-based treatment and behavioral coaching intervention had a positive impact on the reduction of glycated hemoglobin (HbAlc) levels over one year in patients with type II diabetes [28]. Previous studies have already shown that monetary incentives appear to be a strongest motivator for older patients affected by type II diabetes [5]. Nonetheless, there are no clear recommendations to design a mobile service and there is no study available 
to assess whether a system that financially rewards patients could be economically viable. Therefore, our research question is: how to design a mobile service that uses financial incentives to increase patient's compliance?

The rest of the paper proceeds as it follows. In section two, we briefly introduce the recent stream of research concerning financial incentives to increase patient's compliance. In section three we describe our design theory and in section four we illustrate an example to show how our mobile service could be financially viable. Section five addresses legal aspects related to our revenue model and section six describes a business model associated to our service. Finally, section seven and section eight conclude the paper by discussing its limitations and by showing some directions for further investigation.

\section{Literature review}

In this section we briefly highlight few papers related to our study and we underline the knowledge gap.

Therapeutic compliance. The compliance rate of long-term medication therapies can be estimated between $40 \%$ and $50 \%$. The rate of compliance for short-term therapy is much higher (between $70 \%$ and $80 \%$ ), while the compliance with lifestyle changes is the lowest (20\%-30\%) [8]. A systematic review of 102 articles has identified five types of factors, which affect non-compliance: (a) patient-centered, (b) therapyrelated, (c) social and economic, (d) healthcare system, and (e) disease [15].

Design of financial incentives for patient's compliance. The analysis of four systematic reviews of reward-based financing has found that financial incentives targeting recipients of healthcare is effective in the short run for simple and distinct, well-defined behavioral goals, whereas there is less evidence that financial incentives can sustain long-term changes [17]. Since people place more weight on the present than the future costs, a system designed to increase the immediate rewards may influence people's propensity to act, by lowering the social and economic factors that lead to non-compliance. Hence, studies on behavioral economics emphasize (a) the importance of frequent feedback and incentives, (b) the motivational power of lotteries regarding other financial features and (c) the motivation force of anticipated regret [28].

Lottery systems for diabetes management software. Financial incentives for diabetes self-management have only begun to be explored. Individuals expect financial incentives to be a stronger motivation for behavioral change [4], even though incentives for behaviors are preferred for tasks which are considered less challenging [5]. Lottery-based incentives improve monitoring rates among patients with uncontrolled diabetes, and it seems that the smaller expected value lottery (\$1.40/day) is considerably more effective in the post-incentives period than the larger expected value lottery (\$2.80) [25].

\section{Methodology}

Since we did not find a theory to design a system that addresses our research question, we apply design science, which addresses so-called wicked problems and seeks out usefulness, rather than truth [13]. We follow the guidelines of Gregor and Jones [12] to describe the eight components of a design theory. In this section we describe the six core components, whereas in the next two sections we describe the two additional components.

Purpose and scope. The purpose of our mobile service is to increase the therapeutic compliance of patients with type II diabetes. We take into account that there are two sets of users: (a) younger patients, who are more familiar with smartphones and have to plan their adult life taking into account their diabetes, and (b) older patients, who might need assistance to use a smartphone and, after the occurrence of diabetes, need to change a lifestyle, which they have been keeping for several decades.

Constructs. Our design theory has four constructs to describe the system: (1) the short-term and the longterm evolution of the patient's clinical situation, (2) the monetary incentives, (3) the change in the healthcare provider efficiency and (4) the change in the motivation of the patient. The short-term improvement of the patient's condition can be measured by the adherence to medication and the meetings with the healthcare provider, which are reported in the patient's logbook. The sustainable change of the patient can be measured by mobile applications that monitor (a) the level of blood glucose, (b) the level of HbA1c and (c) the Body Mass Index. The monetary incentives are measured by the money delivered to the patient. Finally, the healthcare provider efficiency can be measured by the average amount of hours spent with the patient, whereas patient's motivation can be measured by a survey that assess intrinsic and extrinsic motivation [20].

Functions of the artefact. Figure 1 represents the two functions of the system by using two rectangles. The healthcare provider and the patient affected by diabetes meet to set the goals, in terms of diet, exercise, medications and smoking cessation. We assume that the patient uses a set of devices to automatically collect data every day, whereas we also expect the healthcare provider to spend some time to set up the platform at the beginning. 


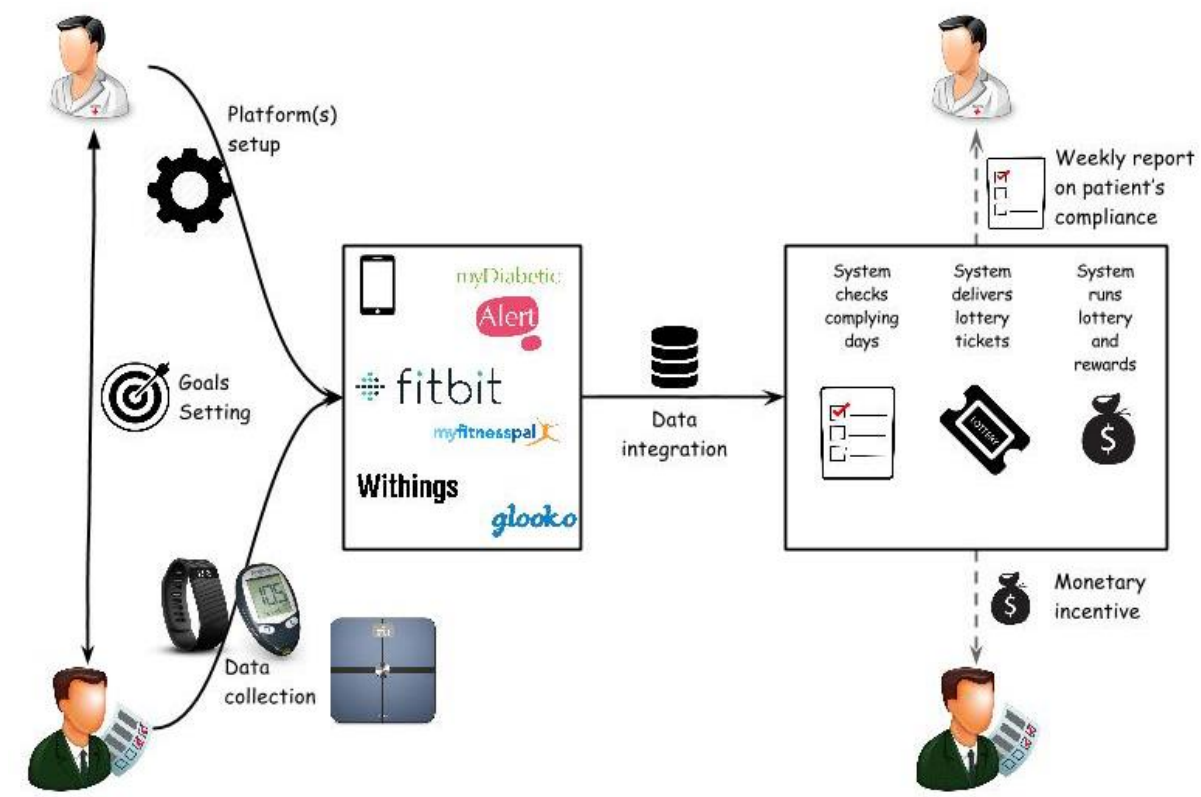

Figure 1: Description of how goals are set, data is analyzed, and rewards are given

This assumption is based on mobile applications like myDiabeticAlert, which contains two roles: patient and healthcare provider. Our system gathers all the information collected by other mobile applications into one single database. Hence, although we consider our service as mobile, we do not create our own mobile application, because we prefer to focus on one complementary component, which can be easily integrated to other applications. Instead, we develop an application that (a) collects data using the Application Programming Interfaces (API) of other mobile applications, (b) checks on the back-end if the data is aligned with the goals, and (c) assigns a lottery ticket to each day the patient is compliant. At the end of each week, a lottery is done and the patient is notified of the result by email.

Kernel theories. Our design theory extends the work about financial incentives for patients affected by type II diabetes [4,5,25].

Testable propositions. By using our four constructs, we derive two sets of propositions. As a starting point, we need to prove that the platform is economically viable without being profitable, due to ethical concerns and legal requirements in some countries. As it turns out, most lottery systems were designed to be fairly profitable and their approach needed to be modified to fulfill our expectations. Therefore, our platform is conceived to assure that compliant participants will receive the service for free, while collecting enough resources to cover operational costs.

P1: Monetary incentives will cover the inscription cost of participants that comply with doctor prescriptions.
We also believe that the random rewards delivered by e-mail will motivate the patients without requiring any additional effort on their side. Moreover, we seek to increase patients' self-management on the long term.

P2: The way monetary incentives are used in the system will increase patient's intrinsic motivation.

Artefact mutability. The system can be customized according to (1) its financial incentives to keep users on the platform and (2) its functionalities. We claim that our system adapts its rewards to let participants shift from extrinsic to intrinsic motivation, following the four steps of the self-determination continuum [23]. We reward every patient, who tries to comply. A patient, who complies only one day each week and gets a single lottery ticket, will win something eventually. This reward will allow passing from external regulation (step 1) to internal regulation (step 2). Nonetheless, the system penalizes the patients, who do not give personal importance to the exercise and comply only to win money. Indeed, if all patients fully comply, they will earn less and less money over time. Therefore, financial incentives over time should be used as a reminder of the ongoing effort, to increase consciousness and awareness and to shift towards identified regulation (step 3 ) and integrated regulation (step 4).

Possible extensions of the lottery system could include gamification and social media components. Nonetheless, most of these requirements are already fulfilled by existing applications for smartphones. 
Table 1: Example of a lottery system auto-financed by 100 patients with type II diabetes

\begin{tabular}{|c|c|c|c|c|}
\hline Variable & Code & Formula & Values & Comments \\
\hline Number of participants & $\mathrm{N}$ & & 10000 & We assume to have 10000 participants \\
\hline Weekly inscription price & WIP & & $\$ 1$ & Each patient pays $\$ 1$ in advance to enter the system \\
\hline Weekly revenues & WR & $\mathrm{N} * \mathrm{WIP}$ & $\$ 10000$ & Every week the system handles $\$ 10000$ \\
\hline Financial support & FSI & & $\$ 0$ & The patient's insurance does not sponsor this system \\
\hline Total revenue & TR & $\mathrm{WR}+\mathrm{FSI}$ & $\$ 10000$ & The weekly revenue depends on the number of participants \\
\hline Profit & $\operatorname{Pr}$ & & $5 \%$ & A percentage of the revenue cover the costs of the platform \\
\hline Total game revenue & TGR & $\mathrm{TR} *(1-\mathrm{Pr})$ & $\$ 9500$ & Every week, $\$ 9500$ are distributed across the winners \\
\hline Patient's compliance & $\mathrm{C}$ & & {$[0-7]$} & Each patient complies somewhere between 0 and 7 days per week \\
\hline Expected Compliance & $\mathrm{EC}$ & & 3 & Each patient is expected to comply 3 days per week on average \\
\hline Winning probabilities & $\mathrm{P}$ & $\mathrm{C} / 7$ & {$[0-100 \%]$} & If the patient complies every day, the system delivers 7 tickets every week \\
\hline Expected winners & EW & $\mathrm{N} * \mathrm{EC} / 7$ & 4300 & The number of winners is assumed to be $10000 * 3 / 7=4286$ \\
\hline Expected Rewards & ER & TGR / EW & $\$ 2.22$ & A participant, who paid $\$ 1$ and complies, should expect to receive $\$ 2.22$ \\
\hline
\end{tabular}

\section{Example of implementation}

In this section, we describe how to implement the lottery function of our system and we offer an illustratory instantiation by means of a basic example.

For sake of simplicity, we assume that the only goal set by the doctor for all the patients is to do 10000 steps per day and that such goal is measured by a Fitbit and shared via its API [11]. In the fourth column of table 1, we assign fictive values to a set of variables, which are listed in the first column and that are needed to assess the revenue model of our service. Table 1 shows how to assess the profitability of the system. In our illustratory example, a winning participant earns up to 2.2 times what was initially spent, whereas the platform can cover its cost to not lose money at the end of the year. Such results can be explained by the fact that each winner receives most of the money from those who did not comply. To confirm our intuition, we wrote a simple script using $\mathrm{R}$ statistical package [22] to perform a simulation that used four matrixes, which had 10000 rows (one for each participant, and 52 columns (one for each week of the year). Simulations are listed among the techniques for artificial evaluations of design research, which allows to assess the performance of the system in different hypothetical conditions [27]. As shown in table 2, the first matrix is PART, which has random numbers between 0 and 7 that simulate the tickets received by the participants. The number 3 implies that the Fitbit data collected from participant P1 shows that she has walked more than 10000 steps three days out of seven, during the first week W1.

The second matrix is ROULE, which has random numbers between 1 and 7 that simulate the individual roulettes. A participant with more tickets has more chances to win, since she is considered to be a winner if the value of PART (her tickets) is equal or greater than the value of ROULE (the outcome of her roulette). The first week (a) P1 lost because she has 3 tickets but the number on her roulette is 4, (b) P3 lost because she needed 6 tickets to win, whereas (c) P2 won because she has 2 tickets and the number on her roulette is 1 .

The third matrix is WIN, which has the list of winners every week. Each week, the system defines the weekly reward by distributing the total amount $(3 * 0.95=2.85$ in the example) among the winners.

The fourth matrix is USD, which multiplies the weekly reward WR by the values of WIN to assess how much each participant has won at the end. The Yearly Reward YR is the sum of the money won each week. In this example, the YR of each participant is slightly below the amount of money that they paid to enter the game ( $\$ 3$ for 3 weeks, whereas the money kept for the platform is $3 * 5 \%=\$ 0.14$ for each participant). 
Table 2: Example with 3 participants over three weeks

(a) weekly compliance of participants

\begin{tabular}{|l|c|c|c|}
\hline PART & W1 & W2 & W3 \\
\hline P1 & 3.00 & 7.00 & 2.00 \\
\hline P2 & 2.00 & 1.00 & 2.00 \\
\hline P3 & 4.00 & 7.00 & 5.00 \\
\hline
\end{tabular}

(b) random results of individual lotteries

\begin{tabular}{|l|c|c|c|}
\hline ROULE & W1 & W2 & W3 \\
\hline P1 & 4.00 & 1.00 & 1.00 \\
\hline P2 & 1.00 & 5.00 & 4.00 \\
\hline P3 & 6.00 & 0.00 & 1.00 \\
\hline
\end{tabular}

(c) weekly winners and weekly rewards

\begin{tabular}{|l|c|c|c|}
\hline WIN & W1 & W2 & W3 \\
\hline P1 & 0.00 & 1.00 & 1.00 \\
\hline P2 & 1.00 & 0.00 & 0.00 \\
\hline P3 & 0.00 & 1.00 & 1.00 \\
\hline WR & 2.86 & 1.43 & 1.43 \\
\hline
\end{tabular}

(d) weekly amount won by each participant

\begin{tabular}{|l|c|c|c|c|}
\hline USD & W1 & W2 & W3 & $Y R$ \\
\hline P1 & 0.00 & 1.43 & 1.43 & 2.86 \\
\hline P2 & 2.86 & 0.00 & 0.00 & 2.86 \\
\hline P3 & 0.00 & 1.43 & 1.43 & 2.86 \\
\hline
\end{tabular}

To test our first proposition, we ran a simulation with random numbers for 10000 participants over 52 weeks. Figure 2 illustrates the distribution of the yearly revenues. The median of our simulated results is close to zero (a participant can expect to receive back the money initially spent). Moreover, figure 2 shows that everyone is expected to win something between $\$ 20$ and \$80. Therefore, proposition P1 (monetary incentives will cover the inscription cost of participants that comply with doctor prescriptions) appears to be validated.

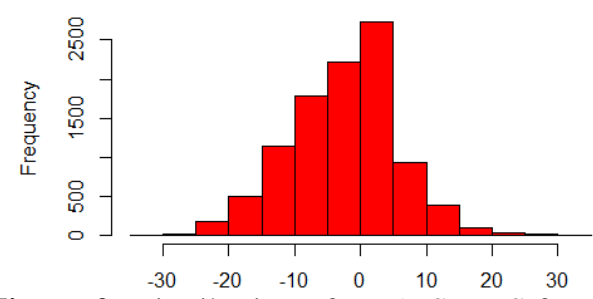

Figure 2: Distribution of $Y R$ (USD VS frequency)

Nonetheless, in our following simulations we took into account that the compliance degree among patients with type II diabetes cannot be expected to be normally distributed, due to the effort required to change the lifestyle of a person.

Therefore, we created a set of matrixes PART with random numbers generated by using a Poisson distribution. Figure 3 shows what would happen with a population of patients that comply less than 2 days per week (lambda $=1$ ), which is compared to a population that complies up to seven days per week (when a simulated participant complies more than 7 days per week, the value is set at 7).

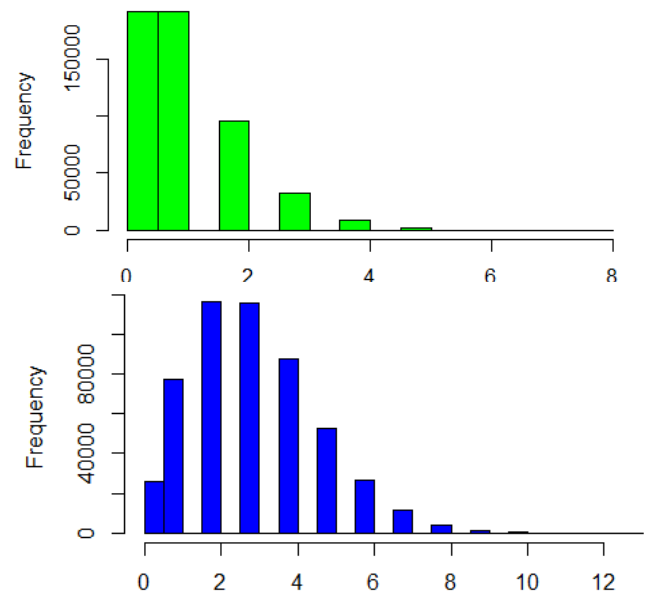

Figure 3: Compliant days VS frequency $(\lambda=1 ; \lambda=3)$

Figure 4 illustrates that, when most people do not comply (lambda=1), those who comply can expect to win a significant amount of money at the end of the year. Nonetheless, as a growing number of participants become motivated to comply (lambda=3), the weekly rewards get smaller and the expected YR gets closer to the one shown in figure 2. Therefore, the system is meant to use extrinsic motivation as an enabler for intrinsic motivation. Therefore, proposition P2 (the way monetary incentives are used in the system will increase patient's intrinsic motivation) appears to be validated.
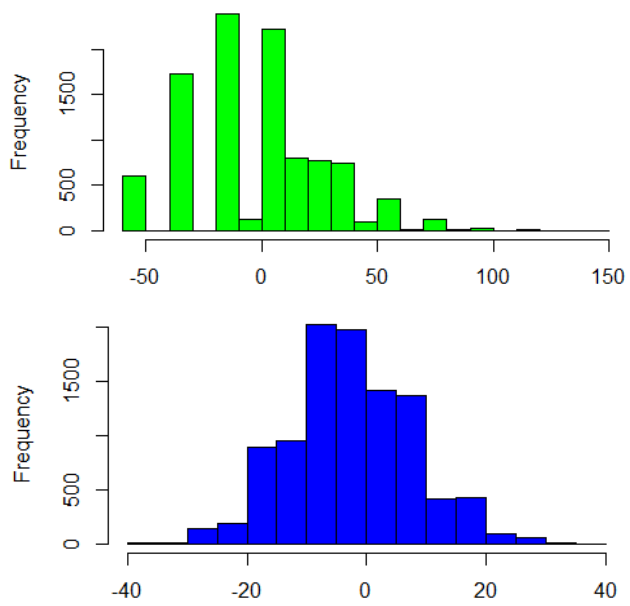

Figure 4: YR: USD VS frequency $(\lambda=1 ; \lambda=3)$ 


\section{Legal aspects}

Since our service will be initially tested in Switzerland, our analysis will focus on legal aspects of our business model with respect to Swiss regulations, which are threefold. Our lottery model (a) is related to an mhealth application, (b) has the possibility to give finance incentives to participants and (c) has not financial activities.

a) Our software is an application for wellbeing. So far, m-health applications are subjected to no specific legislation in Switzerland. However, according to the Directive 93/42/EEC [9] we can use the definition of medical device to claim that our application concerns wellbeing, since it does not give any therapeutic or diagnostic advice.: According to article 1, paragraph 2(a), "'medical device' means any instrument, apparatus, appliance, software, material or other article, whether used alone or in combination, including the software intended by its manufacturer to be used specifically for diagnostic and/or therapeutic purposes and necessary for its proper application, intended by the manufacturer to be used for human beings for the purpose of: (a) diagnosis, prevention, monitoring, treatment or alleviation of disease, $(b)$ diagnosis, monitoring, treatment, alleviation of or compensation for an injury or handicap, (c) investigation, replacement or modification of the anatomy or of a physiological process, (d) control of conception". Therefore, our platform can be classified as a wellbeing application, which falls under the Directive 2011/83/EU [6] on consumer' rights especially in case of distance contracts (art. 2) and data protection (art. 62).

b) Our online lottery system does not seek for profit and aims at common good. The online lottery system in Switzerland is regulated by the article 106, al. 1 of the Federal Constitution, which distinguishes between two kinds of gaming, i.e. casino gambling and betting/lotteries. Our system is related to the Federal Act on Lotteries and Commercial Betting of 8 June 1923 [2], which defines a lottery as "any operation that offers, in exchange for payment or at the conclusion of a contract, the chance to realize a material benefit consisting of a lot, the acquisition, size or nature of this lot being subordinated to, according to a plan, numbers or titles randomly drawn, or to some similar method" (art. 1). Since our model is economically viable but it does not seek for profit, our service falls into the category of lotteries, which benefit charities or the common good and that are allowed by Swiss regulation (art. 3). Indeed, our system respects the following conditions: a) it acts in favor of the general interest, b) it allocates its funds exclusively to the pursuit of these goals, c) exercises actually its activity, d) it targets a broad circle of participants, e) it acts selflessly and $f$ ) it refrains from engaging in commercial activities. Finally, our system will have to comply to the Federal Law on money laundering [3], which imposes the control to the gamer's identity in case of online games (ch.2, sec. 1, art 3).

c) Our lottery is not a financial activity. Our platform does not stock money for its users, and it should not be confused with banking and financial services. Indeed, each participant transfers the money directly to the association at the beginning of the year. Since, the ownership of the money is transferred, our service cannot be qualified as a financial intermediary service according to the Swiss Independent Supervisory Authority for the Financial Markets (FINMA).

\section{Business model of the mobile service}

In this section we illustrate the different components of the business model enabled by our mobile service (figure 5). As mentioned above, our artefact is the design theory describing our lottery system, which has been tested ex-ante by simulation, whereas the business model gives a further view on the expected functionalities and logic of the system. We refer to the eight components of the business model canvas [16], to briefly highlight why our service is expected to be economically viable. We describe a solution for a small team of 5 people; this team could be composed of employees of a hospital, which wants to reduce its hospitalization costs, or it could be part of a private firm that wants to implement a social business model (we do not take into account here the startup that seeks for acquisition by a larger player).

Customer segments. For sake of simplicity, we focus here on the three main customer segments: (a) patients, (b) caregivers/healthcare providers and (c) insurance firms. Patients should be considered both as users and clients. Indeed, they should invest some money in order to be motivated to improve their lifestyle. In our simulation, we have suggested that a community of 10000 patients can be reached, even though that will require a significant amount of effort to promote the service and to manage the community of patients. In comparison, a service such as "Patient like $m e$ " claims to have 380000 patients contributing health data on over 2500 diseases [19]. Therefore, healthcare providers would be key players for the success of the service, by promoting the mobile service to their patients in order to reduce the number of hospitalizations due to lack of compliance. Healthcare providers can choose to receive a report about patients' compliance, as a complement to the information already offered by other applications such as myDiabeticAlert. Finally, insurance firm could decide 
to sponsor this service as a way to reduce the cost of hospitalization, in their own interest and in the interest of their clients. Nonetheless, in this article we assume that the insurance does not pay for this service.

Value propositions. The service offers a new way to use extrinsic motivation to improve the patients' compliance. This idea is presented to patients as a tool that properly rewards their efforts, whereas it is presented to healthcare providers and insurance firm as a way to reduce the costs of multiple hospitalizations.

Customer relationship. The service offers feedback every week to every patient by delivering a reward, even though the interaction with patients is fairly standardized to reduce operational costs and to focus the "job to be done". On the one hand, the interaction with patients and healthcare providers is kept to the minimum in order to not give the impression of an additional application (the overall systems needs to be initially setup and then it automatically collects data and gives updates every week). On the other hand, the interaction with insurance firm is extremely limited due to privacy concerns.

Channels. Patients are reached by online promotion or advised by healthcare providers (for sake of simplicity, we do not take into account patients associations), whereas professional caregivers can be reached with specialized publications and talks at professional gatherings. The service is delivered to patients and healthcare providers by e-mail, as a complement to dashboards already offered by other mobile applications. Insurance firms do not really interact with the system and are most likely interested in presentations about the overall performance of the system.

Key activities. A crucial task for the success of the platform consists in the acquisition and retention of patients, since the simulation has shown how the average cost for each participant gets closer to zero as soon as the degree of compliance among participants resembles to a normal distribution. Hence, marketing efforts and community management have the highest priority as well as the tasks required to assure that the system can automatically collect and analyze the sensor data about each patient.

Key resources. On the one hand, the lottery system and the know-how associated to its way of working are a valuable set of resources. On the other hand, the community of patients and healthcare providers is what keeps the overall service alive. Moreover, we list the patient's data among the key resources, as a reminder of all the privacy concerns associated with medical records. Patients' data is initially collected and stored by other applications and one could decide to erase the data every week, once the winners are declared, as long as there is no need to audit the lottery system afterwards.

Key partners. The patients and healthcare providers are important partners to promote the service among patients; hence they should be considered both partners and customers. Moreover, professional caregivers could take part as medical advisors during the research and development process. Smartphone applications that collect data about the patients are key partners, since they allow reducing the cost for data collection and software development. Nonetheless, the risk associated to outsourcing these tasks is related to the high degree of uncertainty concerning the evolution of the ecosystem of these applications, both in terms of merge and acquisition and in terms of survival rate. This could lead to significant cost for constant adaptation of the interfaces between our platform and the other applications.

Cost structure. A large part of the inscription fees goes back to the patients. The remaining part of the revenues should be used for marketing \& sales and for research \& development. As previously mentioned, our cost structure assumes a small team of five people, which has externalized most of the tasks.

Revenue model. Assuming that the service reaches a community of 10000 participants, one could expect the patients inscription fee to shift from $\$ 1$ per week to $\$ 1$ per day, leading to a revenue flow of some $\$ 180000$ from the $5 \%$ commission rate. Indeed, even though a subscription fee of $\$ 365$ per year might discourage a larger amount of patients, this cost is less than the subscription for a gym and our simulation shows that compliant patients will have most of their money back at the end of the year. If we assume an inscription fee of $\$ 1$ per day, it would be possible to devolve a certain amount of collected money to support social projects, in accordance to Swiss regulations. The revenue flow associated to the doctors might seem reasonable but it will not bring a significant amount of revenues until the system has been tested according to hospital standards. In the first year, some money could come from research projects to assess the feasibility of the model, but this should not be seen as a sustainable solution. Therefore, a partnership with some insurance firms might be required, even though that option brings questions related to privacy concerns and potential conflicts of interest. 


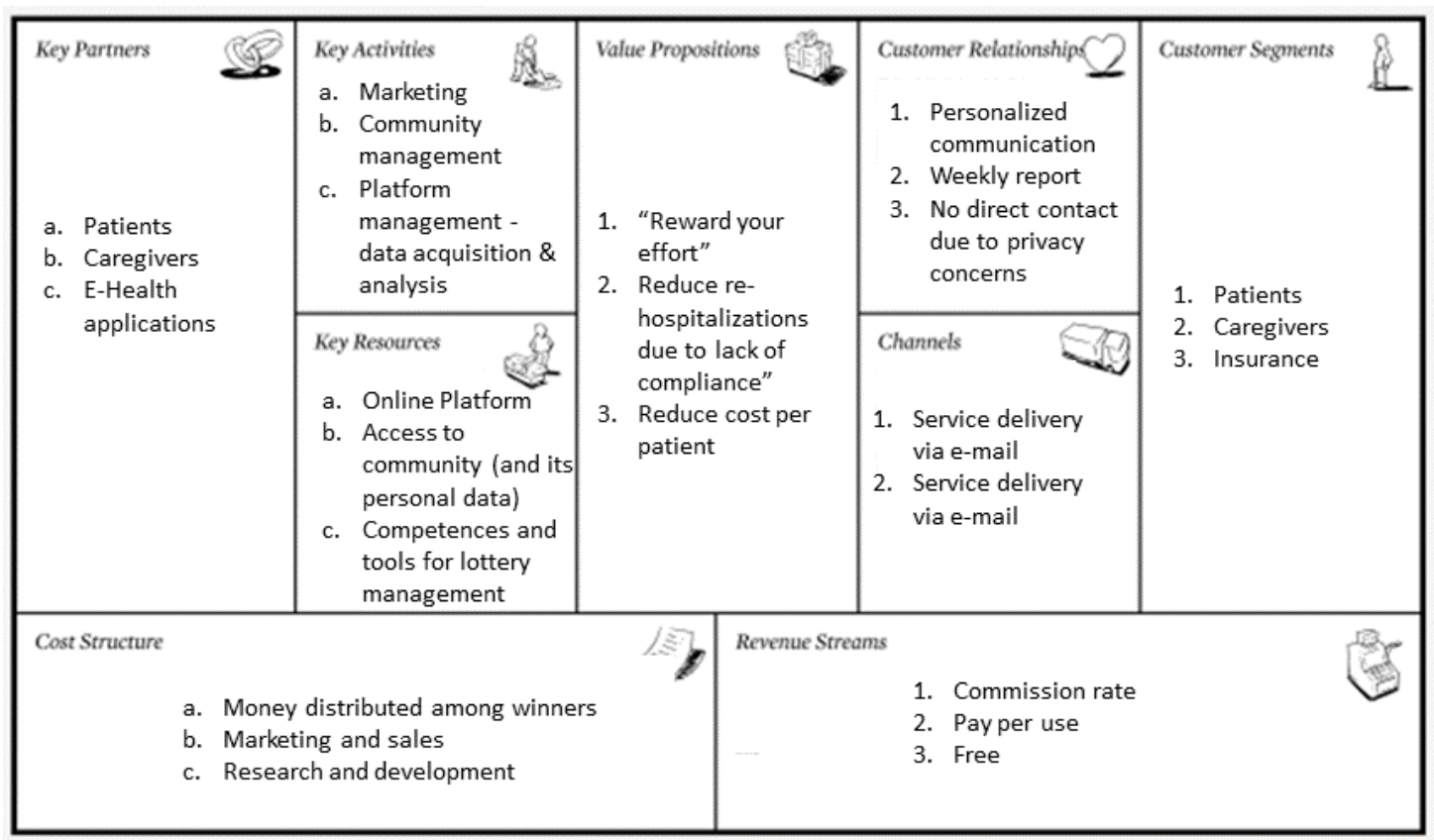

Figure 5: Business model of our mobile service

\section{Discussions}

In this section we address the main remarks received from field experts.

A preliminary work. In this article we describe a design theory [12] and we illustrate an example of implementation by means of a simulation. Artificial evaluation can be seen as unreal due to (a) unreal users, (b) unreal systems, and (c) unreal problems (not held by the users and/or not real tasks, etc.) [26]. Nonetheless, Pries-Heje et al. [21] recommend to use this type of artificial evaluation as an ex-ante assessment of the system, before testing it on larger scale with real users (naturalistic evaluations).

A new way to use patient's data to reduce the cost of multiple hospitalizations. Our approach offers a complementary way to use data from pervasive systems for e-health and it focuses on the decrease in patient's motivation over time, the limited time of professional caregivers to perform a personal followup of each patient and the costs of hospitalizations associated to lack of patient compliance. We move beyond the reliability of data presented by mobile applications, since we consider this data as a feedback for the patient and not a reliable tool for a professional caregiver.

A new set of complex problems to address. Our design theory opens new directions of investigations concerning complex issues such as (1) input: how can healthcare providers set goals that can be measured by smartphones applications? (2) output: how to compare the effect of monetary incentives with respect to traditional solutions already included in mHealth applications? (3) business model: how does the business model change if we take into account the contribution of insurance firms (privacy, revenue model, lottery system)?

\section{Conclusions}

This article illustrates the preliminary results of an ongoing study that describes a new component for diabetes management software, which uses financial incentives to increase patient compliance.

By following the guidelines for a design theory, we describe a system, which combines existing mobile applications to support an economically sustainable lottery with a business model that rewards compliant patients without financial deficit.

Our design theory has been only tested by simulation as an ex-ante assessment and it requires empirical testing to confirm its validity. Nonetheless, we believe that our research opens new interesting directions of investigations to include financial rewards in diabetes management software to improve patient compliance, which may also apply to other chronic diseases other than diabetes.

Acknoledgments: The authors would like to thank Vincent Grèzes for his support while assessing the legal aspects of our lottery system. 


\section{References}

[1] Arnhold, M., Quade, M., and Kirch, W. Mobile applications for diabetics: a systematic review and expert-based usability evaluation considering the special requirements of diabetes patients age 50 years or older. Journal of medical Internet research 16, 4 (2014).

[2] Assemblée fédérale de la Confédération suisse. Loi fédérale sur les loteries et les paris professionnels. 2011.

[3] Assemblée fédérale de la Confédération suisse. Loi fédérale du 10 octobre 1997 concernant la lutte contre le blanchiment d'argent et le financement $d u$ terrorisme (Loi sur le blanchiment d'argent, LBA). .

[4] Blondon, K., Klasnja, P., Coleman, K., and Pratt, W. An exploration of attitudes toward the use of patient incentives to support diabetes selfmanagement. Psychology \& Health 29, 5 (2014), $552-563$.

[5] Blondon, K.S. Patient attitudes about financial incentives for diabetes self-management: A survey. World Journal of Diabetes 6, 5 (2015), 752-758.

[6] Consumer Rights Directive. Directive 2011/83/EU of the European Parliament and of the Council of 25 October 2011 on consumer rights, amending Council Directive 93/13/EEC and Directive 1999/44/EC of the European Parliament and of the Council and repealing Council Directive 85/577/EEC and Directive 97/7. (2011).

[7] Cotterez, A.P., Durant, N., Agne, A.A., and Cherrington, A.L. Internet interventions to support lifestyle modification for diabetes management: a systematic review of the evidence. Journal of diabetes and its complications 28, 2 (2014), 243-251.

[8] DiMatteo, M.R. Patient adherence to pharmacotherapy: the importance of effective communication. Formulary (Cleveland, Ohio) 30, 10 (1995), 596-8.

[9] EC. Council Directive 93/42/EEC of 14 June 1993 concerning medical devices. 1993.

[10] El-Gayar, O., Timsina, P., Nawar, N., and Eid, W. Mobile applications for diabetes self- management: status and potential. Journal of diabetes science and technology 7, 1 (2013), 247-262.

[11] Fitbit.com. Activity \& Exercise Logs - Fitbit Web API Docs. 2016. https://dev.fitbit.com/docs/activity/.

[12] Gregor, S. and Jones, D. The anatomy of a design theory. Journal of the Association for Information Systems 8, 5 (2007), 312-335.

[13] Hevner, A.R., March, S.T., Park, J., and Ram, S. Design science in information systems research. MIS Quarterly 28, 1 (2004), 75-105.

[14] Jiang, H.J., Stryer, D., Friedman, B., and Andrews, R. Multiple hospitalizations for patients with diabetes. Diabetes care 26, 5 (2003), 14211426.

[15] Jin, J., Sklar, G.E., Oh, V.M.S., and Li, S.C. Factors affecting therapeutic compliance: A review from the patient's perspective. Therapeutics and clinical risk management 4, 1 (2008), 269.

[16] Osterwalder, A. and Pigneur, Y. Business Model Generation: A Handbook for Visionaries, Game Changers, and Challengers. John Wiley and Sons, Hoboken, NJ, 2010.

[17] Oxman, A.D. and Fretheim, A. An overview of research on the effects of results-based financing. (2008).

[18] Patel, M.S., Asch, D.A., and Volpp, K.G. Wearable devices as facilitators, not drivers, of health behavior change. Jama 313, 5 (2015), 459-460.

[19] Patients Like Me. About us - Open Research Exchange. Open Research Exchange. https://www.openresearchexchange.com/about.

[20] Pelletier, L.G., Tuson, K.M., and Haddad, N.K. Client motivation for therapy scale: A measure of intrinsic motivation, extrinsic motivation, and amotivation for therapy. Journal of personality assessment 68, 2 (1997), 414-435.

[21] Pries-Heje, J., Baskerville, R., and Venable, J. Strategies for design science research evaluation. ECIS 2008 proceedings, (2008), 1-12.

[22] R. Core Team. R: A language and environment for statistical computing. R Foundation for Statistical Computing, Vienna, Austria, 2013. 
[23] Ryan, R.M. and Deci, E.L. Self-determination theory and the facilitation of intrinsic motivation, social development, and well-being. American psychologist 55, 1 (2000), 68.

[24] Sackett, D.L. and Haynes, R.B. Compliance with therapeutic regimens. (1976).

[25] Sen, A.P., Sewell, T.B., Riley, E.B., et al. Financial Incentives for Home-Based Health Monitoring: A Randomized Controlled Trial. Journal of General Internal Medicine 29, 5 (2014), 770-777.

[26] Sun, Y. and Kantor, P.B. Cross-Evaluation: A new model for information system evaluation. Journal of the American Society for Information Science and Technology 57, 5 (2006), 614-628.

[27] Venable, J., Pries-Heje, J., and Baskerville, R. A comprehensive framework for evaluation in design science research. In Design Science Research in Information Systems. Advances in Theory and Practice. Springer, 2012, 423-438.

[28] Volpp, K.G., Loewenstein, G., Troxel, A.B., et al. A test of financial incentives to improve warfarin adherence. BMC Health Services Research 8, 1 (2008), 272.

\section{Annex 1: The code used for the simulation using $\mathbf{R}$}

\#PARAMETERS
$\mathrm{N}=10000$
$\mathrm{WIP}=1$
$\mathrm{D}=52$
$\mathrm{WR}=\mathrm{N} * \mathrm{WIP}$
$\mathrm{FSI}=0$
$\mathrm{TR}=\mathrm{WR}+\mathrm{FSI}$
$\mathrm{Pr}=0.05$
TGR $=\mathrm{TR} *(1-\mathrm{Pr})$

\#SIMULATION 1: Normal distribution library(truncnorm)

$\mathrm{ECN}=\operatorname{matrix}(\operatorname{rep}(0, \mathrm{~N} * \mathrm{D}), \mathrm{N}, \mathrm{D})$

$\mathrm{EC} 1 \mathrm{~N}=$ matrix $(\mathrm{rep}(0, \mathrm{~N} * \mathrm{D}), \mathrm{N}, \mathrm{D})$

sigma $=1$

$\mathrm{n}=\operatorname{seq}(1: \mathrm{N})$

$\mathrm{d}=\operatorname{seq}(1: \mathrm{D})$

mean $=$ matrix $(\operatorname{rep}(0, N * D), N, D)$

for ( $\mathrm{i}$ in $\mathrm{d})\{$

for $(\mathrm{j}$ in $\mathrm{n})\{$

$\operatorname{set} \operatorname{seed}(\mathrm{j} * \mathrm{i})$

mean $[\mathrm{j}, \mathrm{i}]=\operatorname{sample}(1: 7,1, \mathrm{~T})$

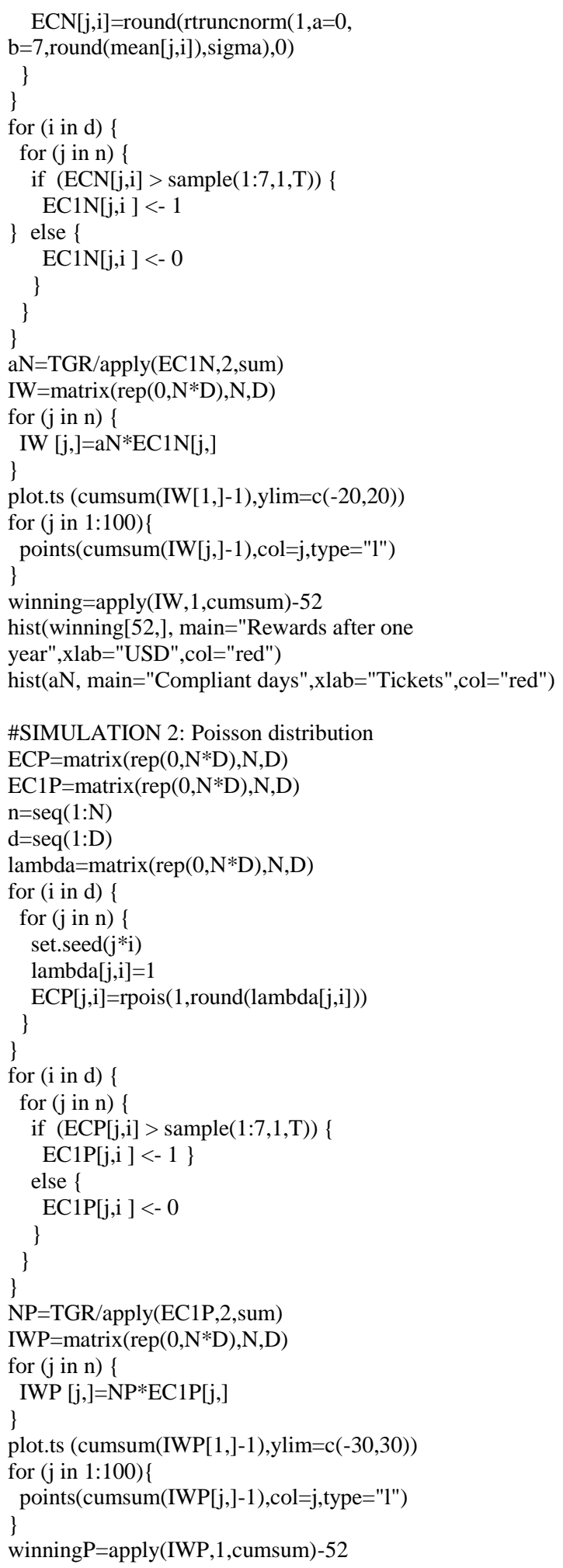

hist(winningP[52,])

hist(winnigP[52,], main="Rewards after one year $($ Lambda=1)

",xlab="Returns",col="green")

hist(ECP, main="Compliant days

(Lambda=1)",xlab="wins",col="green") 